\title{
Experiencias y cambios en los padres de niños con parálisis cerebral infantil: estudio cualitativo
}

\section{Experiences and changes in parents of children with infant cerebral palsy: a qualitative study}

\author{
M. Fernández-Alcántara ${ }^{1}$, M.P. García-Caro ${ }^{2}$, M. Berrocal-Castellano ${ }^{3}$, A. Benítez ${ }^{4}$, \\ C. Robles-Vizcaíno ${ }^{4}$, C. Laynez-Rubio ${ }^{4}$
}

\section{RESUMEN}

Fundamento. El diagnóstico de parálisis cerebral infantil (PCI) supone un evento de carácter traumático que puede provocar una multitud de efectos y cambios en el entorno familiar. Se pretende conocer cuáles son las principales dificultades que encuentran los padres en el proceso de parentalidad, especialmente en los primeros momentos tras el diagnóstico.

Método. Se realizó un estudio fenomenológico de carácter cualitativo a través de entrevistas semi-estructuradas. Participaron 16 padres y madres cuyos hijos estaban diagnosticados de PCI. El análisis de los datos se llevó a cabo con el software Atlas.ti6.2 a través de una codificación abierta.

Resultados. La recepción de la noticia es percibida como un acontecimiento inesperado que obliga a restructurar las expectativas con respecto a su hijo. La forma de relacionarse con el hijo con PCI es diferente a la que se establece con otros niños, centrándose sobre todo en las posibilidades de mejora y en la evolución de su hijo en el futuro. Se observan cambios en diferentes aspectos de la vida de estos padres como el tiempo, la situación económica y laboral, así como las relaciones de pareja.

Conclusiones. En la atención a niños con PCI es necesario incorporar y tomar en cuenta la problemática de los padres, especialmente en los primeros momentos del diagnóstico. El proceso de crianza de un hijo con PCI conlleva un gran número de cambios en la dinámica familiar por lo que se necesita abordarlos mediante una perspectiva global.

Palabras clave. Parálisis cerebral. Padres. Estudio cualitativo. Cuidados familiares.

\begin{abstract}
Background. The diagnosis of infant cerebral palsy (ICP) is a traumatic event that can provoke multiple effects and changes in the family. The aim of the study is to discover the difficulties that parents face in the process of parenting, especially in the initial period following diagnosis.
\end{abstract}

Methods. A qualitative study was carried out through semi-structured interviews. Sixteen mothers and fathers whose children were diagnosed with cerebral palsy participated in the study. Data analysis was performed with Atlas.ti 6.2 software following a strategy of open coding.

Results. The reception of the diagnosis is perceived as an unexpected event that makes parents change expectations and hopes related to their children. The mode of relation with the child with ICP is different from that with other children as parents are more focused on the possibility of improvement and the future evolution of their child. Changes in different aspects of the lives of these parents are shown, such as demands on time, their economic and labour situation, as well as the relationship of the couple.

Conclusions. In providing care for children with cerebral palsy it is necessary to take the problems of the parents into account, especially in the initial period after diagnosis. The process of parenting a child with cerebral palsy entails many changes in the family so a global perspective is needed to organize interventions.

Key words. Cerebral palsy. Parent. Qualitative research. Family caregiving.
1. Dpto. de Personalidad, Evaluación y Tratamiento Psicológico. Facultad de Psicología. Universidad de Granada.

2. Dpto. Enfermería. Facultad de Ciencias de la Salud. Universidad de Granada.

3. Asociación Aderes. Granada.

4. Unidad de Seguimiento y Atención Temprana. Hospital Universitario San Cecilio. Granada.

\section{Correspondencia:}

Manuel Fernández Alcántara

Facultad de Psicología

Universidad de Granada

Campus de Cartuja, s/n

18071 Granada

E-mail: manufer@correo.ugr.es

Recepción: 27 de julio de 2012

Aceptación provisional: 21 de septiembre de 2012

Aceptación definitiva: 2 de octubre de 2012 


\section{INTRODUCCIÓN}

La Parálisis Cerebral Infantil (PCI) es la afección neuromotriz más común en la primera infancia, siendo la principal causa de discapacidad física en niños ${ }^{1,2}$. El nacimiento de un niño con problemas supone un vuelco en la estructura familiar ${ }^{3}$, especialmente cuando la noticia se produce de forma inesperada ${ }^{4}$. Los padres experimentan durante los primeros momentos una serie de emociones complejas y cambiantes, que incluyen el shock, la angustia, la negación o la rabia ${ }^{5}$.

Diversos estudios han puesto de relevancia cómo el nacimiento de un hijo con PCI supone un cambio para los padres en multitud de aspectos de su vida. Guyard y $\operatorname{col}^{6}$ muestran en un trabajo de revisión las principales áreas que se verían afectadas: el tiempo, la situación laboral y profesional, las relaciones sociales, las relaciones familiares, la salud física y psicológica, así como los recursos económicos.

Con respecto al tiempo, parece que criar a un niño con PCI supone una mayor implicación puesto que en muchos casos la independencia que pueden adquirir está muy limitada, necesitando ayuda permanentemente $^{6}$. La situación laboral puede verse alterada debido a la gran necesidad de dedicación al hijo, afectando también a los ingresos económicos de la familia.

Así mismo las madres y padres de niños con PCI parecen experimentar a lo largo del proceso de parentalidad un mayor número de eventos estresantes ${ }^{7}$, que se asocian con un peor pronóstico de salud para las madres ${ }^{8}$. Diferentes estudios han puesto de manifiesto la existencia de altos niveles de estrés en estos padres, en muchos casos alcanzando un nivel clínicamente significativo, requiriendo intervención y apoyo especializado ${ }^{9}$. Algunos autores señalan como posibles causas los comportamientos de los niños, que afectan a áreas como la relación de pareja o el sentido de competencia como padres. También se han asociado a estos padres otros problemas físicos y psicológicos como la aparición de problemas de dolor cervical o de sintomatología ansiosa y depresiva $^{6}$.

Por otra parte, los profesionales de la salud que intervienen en estos casos plantean la necesidad de situar la experiencia de duelo que tiene lugar en los padres $^{7}$. Concretamente hablan de un duelo doble: el duelo por el hijo esperado, que no es el que llega, teniendo los padres que procesar la pérdida del hijo que deberían haber tenido; y el duelo por su propio reconocimiento como padres ${ }^{4,10}$.

Teniendo en cuenta estas especificidades, Almasri y col ${ }^{11}$ proponen un modelo conceptual sobre las necesidades de estas familias, diferenciando tres tipos de áreas: las necesidades relacionadas con el estado de salud del niño, las relacionadas con el funcionamiento familiar, y las relacionadas con el sistema sanitario y los recursos económicos. Destacan también la heterogeneidad en las demandas familiares y la necesidad de un marco abierto que permita una entrada a la subjetividad de cada familia ${ }^{11}$.

El proceso de convertirse en padres de un niño con parálisis cerebral es complejo y trae consigo cambios en diferentes niveles. Es difícil poder planificar una intervención de carácter emocional en estos padres sin conocer cuáles son los principales obstáculos con los que ellos se encuentran, y para descubrir esto, la metodología cualitativa es la más adecuada. No hemos encontrado en la literatura estudios que exploren en profundidad los problemas con los que parecen encontrarse los padres de estos niños, especialmente en los primeros momentos tras el diagnóstico. Por ello, el objetivo de este trabajo fue explorar el discurso y las experiencias de los padres con hijos diagnosticados de PCI en el momento del diagnóstico, así como en los cambios producidos en diferentes aspectos de su vida. Este objetivo se concreta en las siguientes preguntas de investigación:

1. Explorar cómo recibieron la noticia de que su hijo estaba diagnosticado con PCI. 
2. Indagar en la forma de relacionarse con sus hijos, así como las particularidades y diferencias con respecto al proceso de crianza.

3. Conocer en qué se han visto modificados diferentes aspectos de su vida, tomando como ejes los aspectos emocionales, laborales, económicos y la relación de pareja.

\section{MATERIAL Y MÉTODOS}

\section{Diseño}

Se diseño un estudio cualitativo de corte fenomenológico basado en la Teoría Fundamentada ${ }^{12}$ cuyo universo fueron padres y madres de niños diagnosticados con Parálisis Cerebral que recibieran intervención en la Unidad de Seguimiento y Atención Temprana (USAT) del Hospital Clínico San Cecilio de la ciudad de Granada (España).

\section{Participantes}

La muestra estuvo compuesta (Tabla 1) por 5 padres $(31,25 \%)$ y 11 madres $(\mathrm{N}=16)$ de un total de 11 niños con PCI. La media de edad fue de 35,09 años (D.T.=5,04). La edad de los niños osciló entre los 5 meses y los 6 años, con una media de 23,61 meses (D.T.=25,46). La mayor parte procedían de la provincia de Granada, siendo un $68,75 \%$ de diferentes pueblos y el resto de la capital.

Los criterios de homogeneidad fueron ser padres de un niño con un diagnóstico de PCI y que su hijo estuviese recibiendo tratamiento en la USAT. Los criterios de heterogeneidad fueron el sexo, la edad del niño, el nivel de estudios y el lugar de residencia (urbano o rural). Los criterios de inclusión fueron que su hijo estuviese diagnosticado de PCI y que aceptasen participar en el estudio. Los criterios de exclusión fueron no recibir tratamiento en la unidad, otros tipos de patología y no aceptar participar en la investigación.

Todos los niños fueron diagnosticados precozmente de PCI tras el nacimiento. Se controló el tipo y la causa de Parálisis Cerebral, presentando en todos los casos un alto grado de dependencia y necesidad de intervención especializada (Tabla 1).

\section{Instrumentos}

Los datos se recogieron a través de entrevistas semiestructuradas, cuyo guión fue elaborado a partir de la revisión bibliográfica realizada, que permitió establecer los principales temas y preguntas (Tabla 2): la relación que establece con su hijo y sus principales preocupaciones; la experiencia del parto y el diagnóstico; la distribución del tiempo, la situación laboral, así como el apoyo social y el estado de salud.

\section{Procedimiento}

Las entrevistas se realizaron durante los meses de enero a marzo de 2012. Posteriormente se realizó un grupo focal con cuatro de los padres y madres, donde se les expusieron los resultados preliminares con el objetivo de poder triangular la información con los propios participantes, así como profundizar en sus vivencias y necesidades.

Antes de la realización de la entrevista se les explicó a los padres los principales objetivos de la investigación y se les entregó el consentimiento informado. La entrevista fue llevada a cabo siempre por el mismo investigador, procurando que las condiciones de las mismas fuesen similares. Éstas se desarrollaron en un único encuentro con cada entrevistado. Una vez grabadas en audio, las entrevistas fueron transcritas por dos de los investigadores utilizando el software F4. 


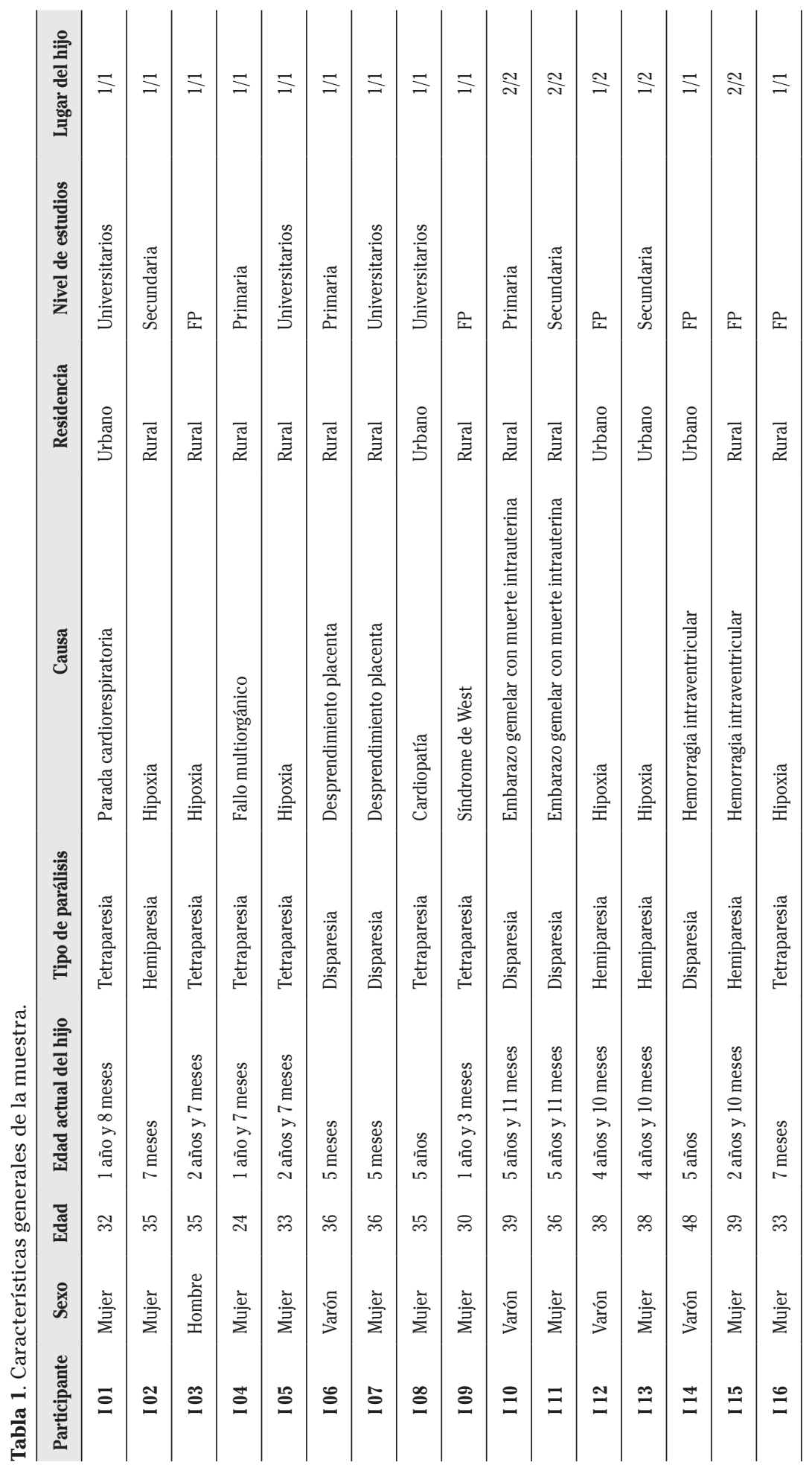


Tabla 2. Estructura del guión de la entrevista semiestructurada.

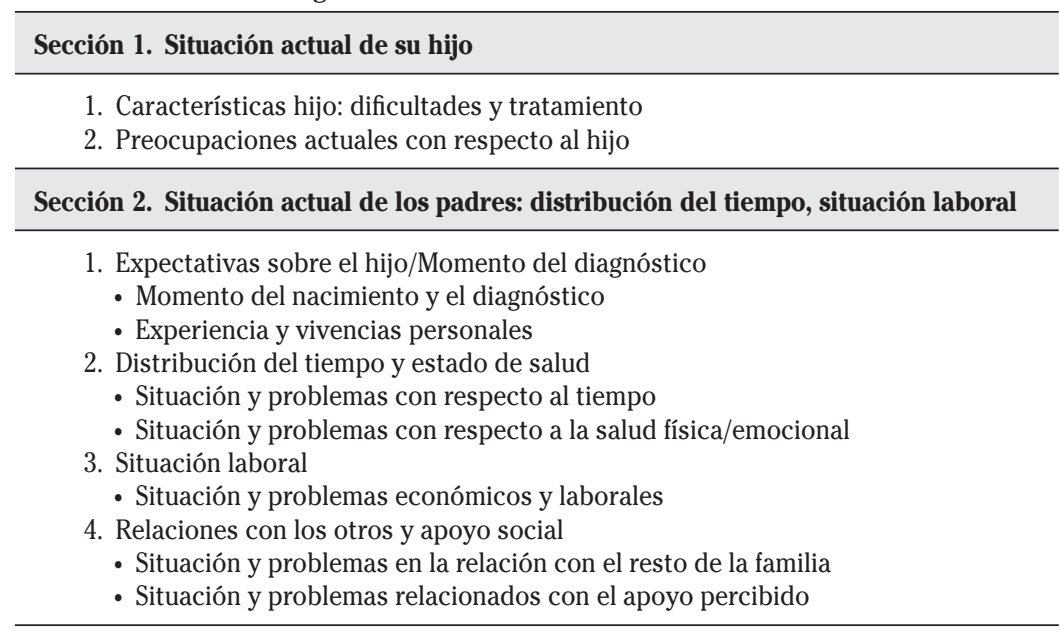

\section{Análisis de los datos}

Para el proceso de análisis e interpretación de los datos cualitativos se ha utilizado el software Atlas.ti en su versión $6.2^{13,14}$, utilizando una estrategia de codificación abierta $^{15}$. El programa Atlas.ti utiliza un sistema de análisis basado en la codificación del texto. En primer lugar se asignan los documentos primarios (entrevistas transcritas) a la base de datos o unidad hermenéutica (HU) creada, para, en segundo lugar, generar las citas o segmentaciones del texto significativas, y codificarlas (asignarles diferentes códigos). Por último, se establecen relaciones (entre códigos o entre códigos y citas), y se crean redes (network) donde se muestra la teoría explicativa a partir del discurso de los participantes ${ }^{16}$.

Para la generación de códigos se han seguido dos pasos diferentes. Por un lado, se ha trabajado con códigos que se han derivado del marco conceptual previo, así como de los objetivos y las diferentes secciones de la entrevista. Por otro, durante el análisis y lectura de las entrevistas se han ido formando nuevos códigos denominados emergentes. Los principales códigos han sido los siguientes: modo de relación con el hijo, dificultades, preocupaciones actuales; problemas y desarrollo del embarazo; experiencia de duelo; cambios en el tiempo, en la situación económica, laboral y de pareja; estado de salud física, emocional y apoyo social.

Para elaborar las redes de relaciones, mediante la herramienta de búsqueda de este programa, es necesario desarrollar un sistema de segmentación del texto en relación a unos códigos genéricos, que han de estar asociados a todas las citas, y unos códigos específicos relativos a la temática concreta del texto.

Los códigos genéricos que se han utilizado han sido la edad, el sexo, el nivel de estudios, la edad del niño y el lugar de residencia. Los códigos específicos son los que se han agrupado en familias de códigos y se muestran especificados más arriba.

Para mejorar la confiabilidad del estudio se controlaron la génesis de los datos, así como la transcripción de las entrevistas. En el primer caso, se ha diferenciado de manera clara entre las citas textuales procedentes de los participantes y las interpretaciones o resúmenes realizados por los investigadores. En el segundo, las entrevistas han sido transcritas por dos de los investigadores, manteniendo unos criterios homogéneos a lo largo de las mismas. Por último, el proceso de análisis y elaboración de los resultados ha estado sometido también a la triangulación entre investigadores para garantizar la credibilidad de los mismos. 


\section{RESULTADOS}

1. Conocimiento del diagnóstico y recepción de la noticia

En los primeros momentos tras el parto lo que aparece en la mayoría de estos padres es un sentimiento de shock e incredulidad. La noticia de que su hijo ha nacido con algún problema resulta imprevista, alterando las expectativas que los padres tienen sobre su hijo, especialmente cuando se trata del primero. En la mayoría de las ocasiones se sienten desbordados y con una gran falta de recursos. Los padres señalan la falta de información con que cuentan por parte del personal sanitario, así como su propia dificultad para asimilarla (Fig. 1).

De manera general, tras conocer la noticia, muchos de los recién nacidos han sido trasladados a la UCI neonatal, lo que supone una gran carga de angustia, tanto por la incertidumbre sobre la evolución del niño, como por el estado en que se encuentra (intubado, con diferentes sondas, etc.,) y por el poco tiempo de que disponen para estar con él (Fig. 1). El tipo de antecedentes o la gravedad del niño no parecen ser factores importantes que medien la vivencia de estas emociones en los padres y madres de este estudio.

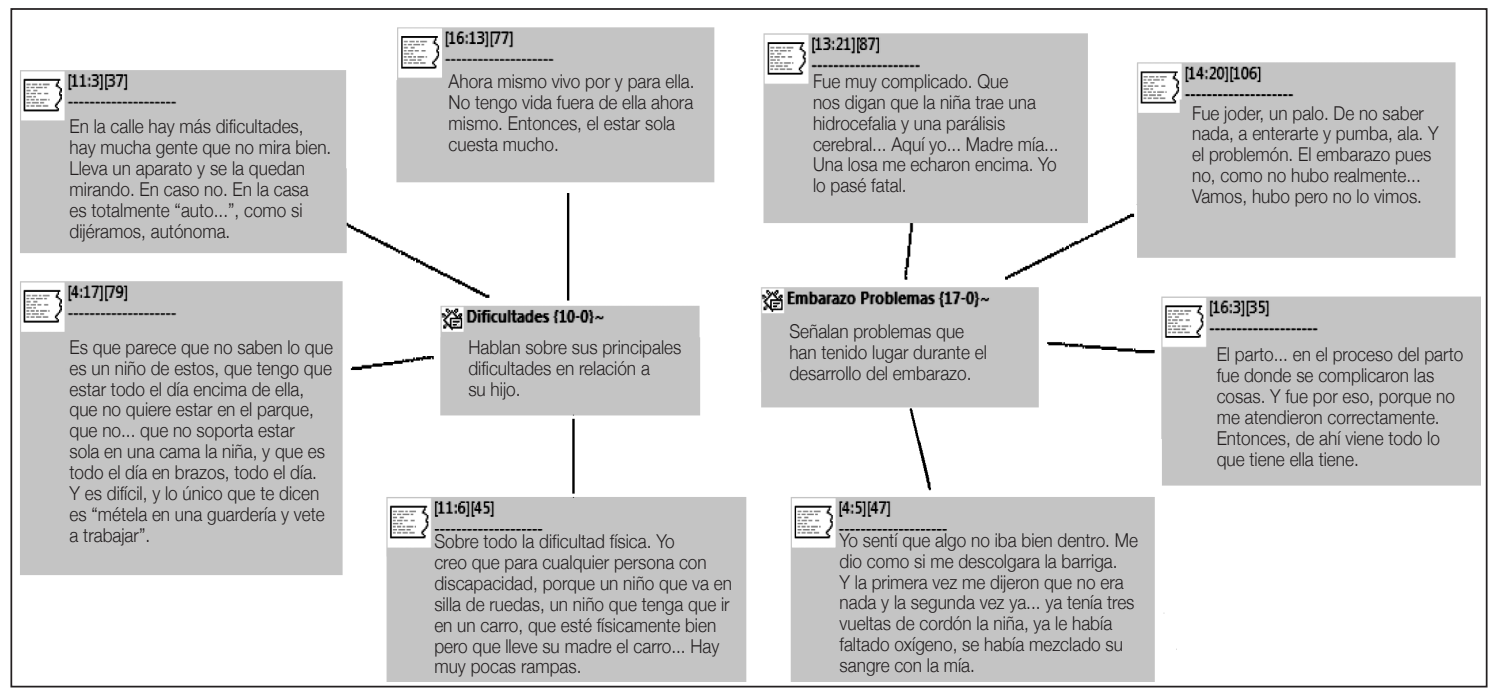

Figura 1. Citas y códigos asociados a los problemas en el parto y a las dificultades con su hijo.

2. Modos de relación, preocupaciones y expectativas con respecto a los hijos

Las relaciones que establecen las madres y los padres con estos niños pueden ser igual a la de otros padres, o estar mediadas por la gran cantidad de cuidado y tiempo que necesitan muchos de ellos. Se requiere un tiempo hasta que los padres pueden adaptarse al niño y a su particularidad. Parece que cuando la pareja tiene otros hijos, o el hijo con PCI es el mayor, el impacto emocional es menor, aunque sigue siendo importante. Aparecen variables me- diadoras como la disponibilidad de tiempo o el trabajo, que influyen en los vínculos que se establecen con el niño (Fig. 2).

Existen un gran número de ámbitos que preocupan a los padres de estos niños, especialmente cómo va a evolucionar su hijo en el futuro. La incertidumbre y las esperanzas que mantienen los padres también se reflejan en el peso que tienen los tratamientos y las intervenciones. Aparece el miedo a no estar haciendo los suficiente, o a que su hijo no evolucione más y mejor por culpa de una falta de atención. En ocasiones los padres 
ponen un gran peso en los tratamientos, quitándole importancia a los momentos de disfrute o juego con su hijo.

Cobra especial importancia que el niño sea capaz de andar y existe una gran preocupación por la autonomía que puedan llegar a alcanzar. En este sentido también muestran una gran inquietud sobre qué pasará cuando ellos ya no estén. Así mismo una de las madres (I 08) señalaba que ella lo que intenta en la actualidad es disfrutar de su hijo conociendo sus limitaciones, pero evitando la angustia permanente por su futuro (Fig. 2).

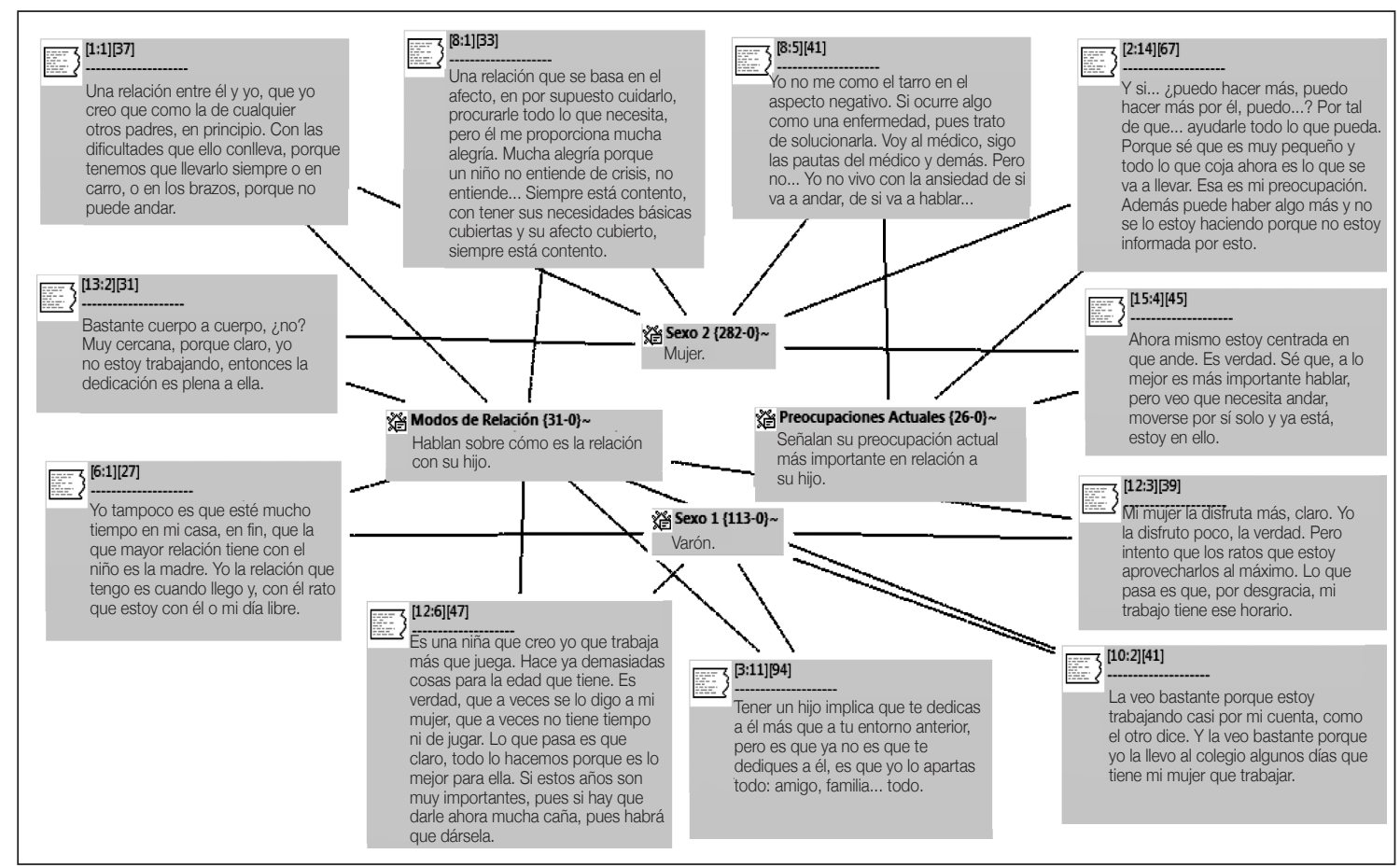

Figura 2. Citas y códigos asociados al modo de relación de los padres con sus hijos y las preocupaciones actuales segmentados según el sexo.

3. Cambios en diferentes aspectos de la vida de los padres

Tras el nacimiento de un hijo diagnosticado con PCI son muchas las áreas donde se producen cambios notables para los padres. Se trata de situaciones que requieren una gran dedicación de tiempo y de esfuerzo físico, lo que afecta a diferentes aspectos de la salud de los padres. En las madres son frecuentes los dolores de espalda, debido al tiempo que pasan con el niño trasladándolo de un sitio a otro. Además, en su opinión enferman con mayor frecuencia que antes de tener a ese hijo (Tabla 3 ).
A nivel emocional estos padres hablan sobre sentimientos de ansiedad y nerviosismo, relacionados con la incertidumbre en la que viven. La experiencia del parto es vivida en muchas ocasiones como un evento traumático que tiene una gran dificultad para procesarse. Algunas madres señalan la importancia que ha tenido para ellas el apoyo familiar, así como el apoyo por parte de profesionales de la psicología, puesto que les han permitido elaborar parte de lo ocurrido, de manera que ahora se relacionan mejor con su hijo y con ellos mismos (I 08). Sin embargo, matizan que es 
muy importante la sensibilidad de los profesionales al abordar temas emocionales relacionados con su hijo. También hay que destacar la posición de algunos hombres que afirman que su salud no se ha resentido en ningún sentido (I 06, I 10, I 12).

Otras áreas que se ven afectadas son el tiempo, la situación económica y laboral, así como las relaciones de pareja. Con respecto al tiempo las madres señalan que el tiempo que existe es el de su hijo y no el suyo. El tiempo pasa muy rápido y además los padres acuden en muchas ocasiones a asociaciones u otros lugares donde el hijo recibe más tratamiento o realiza actividades complementarias de rehabilitación (Tabla 3).

Tabla 3. Cambios en relación a la salud física y emocional, al tiempo, a la situación económica, laboral y de pareja

\begin{tabular}{|c|c|}
\hline Códigos & Citas \\
\hline \multirow[t]{3}{*}{$\begin{array}{l}\text { Situación } \\
\text { económica y } \\
\text { laboral }\end{array}$} & $\begin{array}{l}\text { "Económicamente se necesitan un montón de apoyos que no existen. Pero un montón. } \\
\text { Hasta yo, que tengo un trabajo indefinido y que lo que cobro está bien, pues necesito más" } \\
\text { (I } 08 \text { - Madre) }\end{array}$ \\
\hline & $\begin{array}{l}\text { "Ahora mismo hay una asociación que me da alimentos y mi madre me ayuda con los gastos } \\
\text { de la niña. Ahora mismo no tengo ningún tipo de ingresos y yo necesitaría algún tipo de } \\
\text { ingresos, por lo menos que me ayudaran con los gastos de la niña y el desplazamiento hasta } \\
\text { aquí" (I } 16 \text { - Madre) }\end{array}$ \\
\hline & $\begin{array}{l}\text { "Me he quitado de trabajar un día en semana, los viernes me los he quitado porque prefiero es- } \\
\text { tar con mi hijo. Económicamente, pues una reducción, ¿no? Pero no me importa" (I } 15 \text { - Madre) }\end{array}$ \\
\hline \multirow[t]{3}{*}{ Tiempo } & $\begin{array}{l}\text { "El tiempo, todo el que tengo, se lo dedico todo a ella. Es lo único que hago. Desde por la ma- } \\
\text { nana a la noche es lo único que hago: dedicarme a ella 100\%. No hago otra cosa" (I } 16-\text { Madre) }\end{array}$ \\
\hline & $\begin{array}{l}\text { "Mi mujer la disfruta más, claro. Yo la disfruto poco, la verdad. Pero intento que los ratos } \\
\text { que estoy aprovecharlos al máximo. Lo que pasa es que, por desgracia, mi trabajo tiene ese } \\
\text { horario" (I } 12 \text { - Padre) }\end{array}$ \\
\hline & $\begin{array}{l}\text { "No creo que ninguna madre que tenga esta situación tenga tiempo. Tiene que ser más o } \\
\text { menos ya cuando el niño está grande, que la cosa siga mal igual, que es lo que yo veo con } \\
\text { otras madres, como otra de las madres. Que el niño sigue mal igual, pero por lo menos ya lo } \\
\text { puede meter en un cole" (I } 09 \text { - Madre) }\end{array}$ \\
\hline \multirow[t]{3}{*}{$\begin{array}{l}\text { Relación de } \\
\text { pareja }\end{array}$} & $\begin{array}{l}\text { "Cuándo me quedé embarazada él me dijo que o la niña, vamos el bebé o él, y yo pues yo } \\
\text { estaba embarazada ya de un mes y pico y yo porque en teoría la niña o yo, pues mira, la niña } \\
\text { está dentro de mi ya, y yo no voy a matar a una niña, porque hubiera sido matar a una niña. } \\
\text { Y lo dejamos cuando nació" (I } 04 \text { - Madre) }\end{array}$ \\
\hline & $\begin{array}{l}\text { "Su papá, mi marido, ha estado siempre al cien por cien. Hemos estado los dos ahí, y mi } \\
\text { familia y su familia" (I } 02 \text { - Madre) }\end{array}$ \\
\hline & "Mi hija es la única persona que me interesa ahora mismo" (I 16 - Madre) \\
\hline \multirow[t]{2}{*}{ Salud emocional } & $\begin{array}{l}\text { "Yo estoy todo el día con la lágrima fácil. Pues eso, con la lágrima fácil. Y ya está. Pues ya } \\
\text { está, si es que no... Porque soy feliz, pero no es la felicidad completa" (I } 15 \text { - Madre) }\end{array}$ \\
\hline & $\begin{array}{l}\text { "Yo padezco cierta ansiedad que yo antes no la conocía, ¿no?, porque con las preocupacio- } \\
\text { nes de la chica pues... Cuando no es una del pie, es la mano, cuando no pues... Hace poco le } \\
\text { diagnosticaron una epilepsia, entonces pues, siempre tienes como muchos frentes abiertos y } \\
\text { el estado de preocupación y de ansiedad y de nervios es continuo. Porque nunca sabes qué } \\
\text { es lo que va a aparecer" (I } 13 \text { - Madre) }\end{array}$ \\
\hline \multirow[t]{2}{*}{ Salud física } & $\begin{array}{l}\text { "Desde que he dejado al niño en la guardería estoy todo el día resfriada, cada dos por tres } \\
\text { con problemas de estómago y con vómitos" (1 } 01 \text { - Madre) }\end{array}$ \\
\hline & $\begin{array}{l}\text { "Estoy siempre mala, siempre... lo pillo todo, todo, que si gripe, que si resfriado, que si... } \\
\text { un virus, que si estoy vomitando. Sí, la verdad es que estoy peor, peor que cuando estaba } \\
\text { trabajando, y me organizo menos, no sé por qué, no sé si es por... agotamiento o los nervios, } \\
\text { que me salen por ahí, por la enfermedad" (I } 05 \text { - Madre) }\end{array}$ \\
\hline
\end{tabular}


En el ámbito laboral se producen modificaciones que dependen mucho de la situación de la pareja. En ocasiones la madre deja de trabajar para dedicarse de manera exclusiva al niño, o reduce su jornada de trabajo. Algún miembro de la pareja tiene que ceder para poder dedicarle todo el tiempo que necesita el niño, algo que se puede compaginar con alguna ayuda puntual, cuando disponen de suficientes ingresos como para contratarla.

Los padres y madres también señalan la importancia del trabajo como un mecanismo de afrontamiento que les permite encontrar otros espacios por fuera de la relación con su hijo. Las relaciones de pareja también pueden verse afectadas, aunque los padres señalan que depende mucho de cómo se encontrasen antes del nacimiento del niño: si la relación ya era complicada, tras el nacimiento del niño los problemas aumentan, por el contrario si la relación era buena por lo general los padres mantienen una buena relación.

\section{DISCUSIÓN}

En este estudio se muestran las principales vivencias de padres y madres que conviven con un hijo diagnosticado de PCI. A pesar de la amplia literatura existente en parálisis cerebral, éste es de los pocos estudios que aborda de manera cualitativa las experiencias de los padres durante los primeros años de vida del niño, periodo en el que el impacto de la noticia es reciente.

Cabe destacar asimismo, que se ha contado con la participación de padres y madres de manera individualizada, independientemente de que fuesen los progenitores del mismo niño con PCI. En nuestro estudio, padres y madres muestran diferencias en su discurso con respecto a los problemas y las experiencias de cada cual con su hijo, aun cuando éste sea el mismo. Por tanto, lo que en este trabajo se expone es la particularidad de cada uno de los progenitores en su relación con el niño. En la literatura científica, se encuentran también diferencias entre padres y madres en situaciones como el duelo perinatal ${ }^{17}$, la pérdida de un hijo ${ }^{18} \mathrm{o}$ el diagnóstico de enfermedad mental ${ }^{19}$.
Las emociones que acompañan el proceso de crianza coinciden con las señaladas en la literatura existente ${ }^{5,11}$, destacando especialmente la aparición de angustia en los primeros momentos tras el parto. Aunque se han observado emociones complejas y estresantes en prácticamente todos los padres y madres participantes, el shock y la incredulidad ${ }^{20}$ parecen ser mayores cuanto menor es la edad del niño. Sin embargo, en este sentido hay que tener en cuenta que, en algunos casos, no es tanto efecto del tiempo cronológico transcurrido sino de las intervenciones emocionales y del trabajo de elaboración de las mismas por parte de los padres.

Muchos de los participantes plantean directamente la necesidad de una intervención psicológica para afrontar la situación mientras, por otra parte, normalizan la experiencia, poniendo así en evidencia las contradicciones emocionales de una experiencia que, si bien es traumática, supone el inicio de su función como padres y de la relación con el hijo. Este conflicto emocional conlleva la separación entre las emociones y el ejercicio de sus obligaciones como padres, lo que parece repercutir en una mayor dificultad para elaborar la pérdida con respecto al hijo ideal ${ }^{7,21}$, por lo que se produciría una adaptación sin tiempo para elaborar los diferentes aspectos emocionales $^{4,10}$. En este sentido, la mayoría de los estudios basados en intervenciones en PCI, destacan la necesidad de incluir la experiencia de la familia, como el caso de los llamados "family-centredservices" 22,23 .

Los padres también han señalado las consecuencias de criar a un niño con PCI, siendo lo más destacable la falta de tiempo que encuentran, así como los efectos que tiene sobre su vida social, laboral y económica. Es interesante notar que muchas de las madres han señalado que a pesar de existir cambios importantes en su modo de vida, están dispuestas a hacer cualquier cosa por sus hijos. Esto es más destacable en el aspecto económico y laboral donde el niño se convierte en una prioridad a pesar de que ciertos tratamientos o intervenciones supongan un gran coste para la economía familiar. 
El apoyo social por parte de la familia parece ser básico, aunque en muchos casos han aparecido problemas de comunicación con otros familiares, ya que éstos no conocen el diagnóstico del niño ni sus particularidades, al menos de manera explícita, por la angustia que les supone a los padres hablar sobre la parálisis cerebral.

La percepción subjetiva sobre su estado de salud se ha centrado sobre todo en la existencia de limitaciones físicas por el peso del niño, así como por una mayor sensibilidad a enfermedades, lo que concuerda con estudios como el de Tong y coll $^{24}$ donde se señalaba la mayor probabilidad de las madres de estos niños de padecer dolores a nivel dorsal.

Los padres y madres que han participado en el estudio afirman dedicar gran parte del tiempo a los cuidados de su hijo. En este caso se unen a las demandas de un niño de corta edad las dificultades específicas que presentan estos niños con respecto a su autonomía y las emociones tan complejas por las que pasan los padres. Diferentes estudios han mostrado esta gran dedicación de tiempo, especialmente en las madres, así como las relaciones entre el tiempo dedicado y los problemas emocionales ${ }^{25}$.

Así mismo, se encuentran muy centrados en que el tiempo que pasan con su hijo pueda ser utilizado como rehabilitación, o como lugar donde ensayar y practicar lo aprendido en la intervención con la fisioterapeuta. Esta necesidad legítima, en ocasiones, no parece estar en consonancia con las necesidades que expresan niños más mayores con parálisis cerebral. El estudio de Parkinson, Rice y Young ${ }^{26}$ muestra cómo para los adolescentes con parálisis cerebral el ambiente en casa era el lugar idóneo para descansar y disfrutar de actividades lúdicas después de un día de tratamientos o esfuerzos, mientras que los padres, si bien consideraban el hogar como el lugar adecuado para que los niños se relajasen, no pensaban que pudiera ser algo especialmente placentero para ellos. Estos padres parecen experimentar una dificultad para acompañar a sus hijos en esos momentos de disfrute, especialmente si no implican algún tipo de estimulación o de interven- ción sobre las habilidades cognitivas o motoras. Parece que en los primeros momentos la adaptación y el cuidado del niño son el eje central sobre el que giran sus padres, ocupando los aspectos emocionales un lugar más secundario, que se va retomando cuando va pasando el tiempo. Es interesante notar que en adolescentes con parálisis cerebral la relación con sus padres cobra una importancia central con respecto a su percepción de la calidad de vida ${ }^{26}$.

En el estudio de Whittingham y $\mathrm{col}^{7}$ los padres señalaron que el saber manejar las conductas de los niños era de gran importancia, y que tenían dificultades para interpretar su comportamiento. En este estudio, al tratarse de niños de corta edad, los padres han mostrado una mayor preocupación por otros aspectos, como su relación de pareja, la evolución de su hijo en el futuro, o la manera de comunicar a otros familiares el diagnóstico ${ }^{7}$. Finalmente, los resultados obtenidos hay que entenderlos dentro del contexto en el que se ha realizado la investigación. Así, futuros trabajos podrían ir orientados a ampliar la muestra a padres de niños con PCI que no estén recibiendo tratamiento en atención temprana, a comprobar si estas preocupaciones se ven modificadas tras la realización de una intervención donde permitan abordarse estas temáticas con los padres, o a profundizar en los efectos de este tipo de relación en adolescentes con parálisis cerebral.

En conclusión, los profesionales de la salud que atienden a los niños con PCI han de integrar y tener en cuenta la problemática de los padres en la intervención rehabilitadora, especialmente en los primeros momentos, donde los padres se ven enfrentados a una gran cantidad de información emocional que puede sobrepasarlos. Es necesario escuchar y atender la perspectiva de los padres y considerar sus experiencias para ajustar y mejorar las intervenciones. El proceso de crianza de un hijo con PCI conlleva un gran número de cambios en la dinámica familiar, por lo que se necesita abordarlos a través de una perspectiva global. Además, es necesario tener en cuenta los modos de relación que 
se establecen con el niño para propiciar un ambiente lúdico y no sólo de trabajo entre los padres y el niño.

Por último, parece que existe dificultad en el sistema sanitario para identificar a los padres de niños con parálisis cerebral como cuidadores, similares a los cuidadores informales de ancianos, de manera que quedan excluidos de los programas específicos para cuidadores de personas dependientes que existen en algunas instituciones. Nuestros resultados muestran en estos padres un importante rol como cuidadores y terapeutas en la relación con sus hijos. Sin embargo, no existen medios para realizar una intervención protocolizada donde las problemáticas de los padres se puedan abordar, y que pueda servir como un espacio donde compartir experiencias.

\section{Agradecimientos}

Nos gustaría agradecer a todos los padres y madres que han colaborado de manera desinteresada en este estudio.

\section{BIBLIOGRAFÍA}

1. Madrigal-MuÑoz A. Familias ante la parálisis cerebral. Intervención Psicosocial 2007; 16: 55-68.

2. Robles-Vizcaíno C, Ocete-Hita E, Benítez-Feliponi A, Ruiz-Extremera A. Parálisis Cerebral Infantil. En: Ruiz-Extremera A, Robles-Vizcaíno C, editores. Niños de Riesgo. Programas de Atención Temprana. Madrid: Norma-Capitel, 2004: 289-300.

3. De Linares C, Rodríguez-Fernández T. Bases de la intervención familiar en atención temprana. En: Pérez-López J, Brito de la Nuez AG, editores. Manual de Atención Temprana. Madrid: Ediciones Pirámide, 2004: 333-354.

4. CRuz F, LaYnez C. Intervención psicológica centrada en la familia: las vivencias de los padres. De la vivencia a la representación. En: Ruiz-Extremera A, Robles-Vizcaíno C, Ocete-Hita E, editores. Temas Emergentes en Neonatología. Granada: Alhulia, 2000: 179188.

5. Sen E, Yurtsever S. Difficulties experienced by families with disabled children. J Spec Pediatr Nurs. 2006; 12: 238-252.

6. Guyard A, Fauconnier J, Mermet MA, Cans C. Impact sur les parents de la paralysie cérébrale chez l'enfant: revue de la littérature. Arch Pediatr 2010; 18: 204-214.

7. Wittingham K, Wee D, Sanders M, Boyd R. Responding to the challenges of parenting a child with cerebral palsy: a focus group. Disabil Rehabil 2011; 33: 1557-1567.

8. Rentinck ICM, KetelaAr M, Jongmans MJ, Gorter JW. Parents of children with cerebral palsy: a review of factors related to the process of adaptation. Child Care Health Dev 2006; 33 : 161-169.

9. Parkes J, Caravale B, Marcelli M, Franco F, ColVER A. Parenting stress and children with cerebral palsy: a European cross-sectional study. Dev Med Child Neurol 2011; 53: 815-821.

10. Cruz F, Laynez C, Ruiz A, Jaspez C. Prematuridad, relaciones familiares y hospitalización. Scientia 1995; 1: 24-28.

11. Almasri Na, Palisano RJ, Dunst CJ, Chiarello LA, O'NeIll ME, Polansky M. Profiles of needs of families of children and youth with cerebral palsy. Child Care Health Dev 2011; 40: 130-154.

12. Glaser BG, Strauss AL. The discovery of Grounded Theory: Strategies for qualitative research. 1sted. Nueva York: Aldine; 1967.

13. Lewis B. NVivo 2.0 and Atlas.ti 5.0: A comparative review of two popular qualitative dataanalysis programs. Field Meth 2004; 16: 439469.

14. Scientific Software Development. Atlas.ti Scientific Software.[consultado 22-07-2012]: Disponible en http://www.atlasti.com/es/

15. Strauss AL, Corbin J. Basics of qualitative research: techniques and procedures for developing grounded theory. 3rd. London: SAGE Publications; 2007.

16. Munr T. Atlas.ti- A prototype for the support of text interpretation. Qual Soc 1991; 14: 349371.

17. Fernández-Alcántara M, Cruz-Quintana F, PérezMarfil N, Robles-Ortega H. Factores psicológicos implicados en el duelo perinatal. Index Enferm 2012; 21: 48-52.

18. Bosquet-Del Moral L, Campos-Calderón C, Hueso-Montoro C, Pérez-Marfil N, Hernández-MoliNERo Á, Arcos-Ocón L et al. Vivencias y experiencias de duelo en madres tras un proceso de enfermedad oncológica de sus hijos. Med Pal 2012; 19: 64-72.

19. Richardson M, Cobham V, Murray J, McDermott B. Parents' grief in the context of adult child mental illness: a qualitative review. Clin Child Fam Psychol Rev 2011; 14: 28-43.

20. Piggot J, Paterson J, Hocking C. Participation in home therapy programs for children with 
cerebral palsy: A compelling challenge. Qual Health Res 2002; 12: 1112-1129.

21. Wittingham K, Wee D, Sanders M, Boyd R. Predictors of psychological adjustment, experienced parenting burden and chronic sorrow symptoms in parents of children with cerebral palsy. Child Care Health Dev [En prensa].

22. Dirks T, Hadders-Algra M. The role of the family in intervention of infants at high risk of cerebral palsy: a systematic review. Dev Med Child Neurol 2011; 53 (Supl. 4): 62-67.

23. Jeglinsky I, Autti-Rämö I, BRogren-Carlberg E. Two sides of the mirror: parents' and service providers' view on the family-centredness of care for children with cerebral palsy. Child Care Health Dev 2011; 38: 79-86.
24. Tong HC, Haig AJ, Nelson VS, Yamakawa KS, Kandala G, Shin KY. Low back pain in adult female caregivers of children with physical disabilities. Arch Pediatr Adolesc Med 2003; 157: 1128-1133.

25. Sawyer MG, Bittman M, La Greca AM, Crettenden A, Borojevic N, Raghavendra P et al. Time demands of caring for people with cerebral palsy: what are the implications for maternal mental health? Dev Med Child Neurol 2011; 53: 338-343.

26. PARKInson KN, Rice H, Young B. Incorporating children's and their parents' perspectives into condition-specific quality-of-life instruments for children with cerebral palsy: a qualitative study. Value Health 2011; 14: 705711. 